\title{
State-dependent delay in regenerative turning processes
}

\author{
Tamás Insperger · Gábor Stépán · Janos Turi
}

Received: 14 October 2005 / Accepted: 24 January 2006 / Published online: 21 November 2006

(C) Springer Science + Business Media B.V. 2006

\begin{abstract}
Stability of a two degrees of freedom model of the turning process is considered. An accurate modeling of the surface regeneration shows that the regenerative delay, determined by the combination of the workpiece rotation and the tool vibrations, is in fact statedependent. For that reason, the mathematical model considered in this paper is a delay-differential equation with state-dependent time delay. In order to study linearized stability of stationary cutting processes, an associated linear system, corresponding to the statedependent delay equation, is derived. Stability analysis of this linear system is performed analytically.

A comparison between the state-dependent delay model and the previously used constant or timeperiodic delay models shows that the incorporation of the state-dependent delay into the model slightly affects the linear stability properties of the system in certain parameter domains.
\end{abstract}

Keywords Machine tool chatter - Regenerative effect $\cdot$ State-dependent delay $\cdot$ Linearization . Stability

\footnotetext{
T. Insperger $(\varangle) \cdot$ G. Stépán

Department of Applied Mechanics, Budapest University of Technology and Economics, H-1521 Budapest, Hungary e-mails: inspi@mm.bme.hu, stepan@mm.bme.hu

J. Turi

Programs in Mathematical Sciences, University of Texas at Dallas, Richardson, TX 75083

e-mail: turi@utdallas.edu
}

\section{Introduction}

Systems governed by delay-differential equations (DDEs) often appear in different fields of science and engineering. One of the most relevant mechanical applications is the cutting process dynamics. An important phenomenon that limits the productivity of machining is the development of self-excited vibrations, also known as machine tool chatter. The work of Tlusty [1] and Tobias [2] led to the development of the regenerative machine tool chatter theory. The basis of regenerative cutting model is that either the tool, or the workpiece or both are flexible and the chip thickness varies due the relative vibrations of the tool and the workpiece. The tool cuts the surface that was formed in the precious cut, and the chip thickness is determined by the current and a previous position of the tool/workpiece. In standard models appearing in the literature, the time delay between two succeeding cuts is considered to be constant, which is equal to the period of the workpiece rotation in turning, or to the tooth passing period in milling. The corresponding mathematical model of the turning process in that case is an autonomous DDE. Models describing milling are DDEs with time-periodic coefficients. DDEs have infinite dimensional state spaces, therefore closed form stability criteria are not available. Stability analysis of these systems is often performed by numerical simulations (see, e.g., [3, 4]), and different "semi-analytical" approximation techniques (see, e.g., [5-13]). 
Models with constant time delay capture the main character of regenerative dynamics, and can be used to describe linear stability properties in good agreement with experiments. However, some phenomena can only be explained using more sophisticated models that incorporate varying time delay. Long and Balachandran $[14,15]$ pointed out that, in an accurate model of the milling process, the regenerative delay is timedependent due to the feed rate and the consequent trochoidal path of the teeth. The resulting model is a DDE with periodic coefficients and with time-periodic delay. They showed that for large feed rates, the traditional constant delay models overestimate stability limits. Faassen et al. [16] used time-periodic delay with an updated model of the undeformed chip thickness and showed that stability boundaries obtained by their new model differ from the ones of traditional models especially for small radial depths of cut.

Time periodic delays also arise in the model of turning with varying spindle speed (see, e.g., [17-19]). The aim of the varying spindle speed technique is to suppress regenerative chatter. The corresponding model equation is a DDE with time-periodic delay. Stability analysis of systems with periodic delay is more complicated than that of systems with constant time delay, still there are numerical algorithms that can be used to perform these calculations. An efficient technique is the semi-discretization method (see [20, 21]), as it was shown in [19] for turning with varying spindle speed.

If the regeneration process is modeled accurately, then the vibrations of the tool should also be included in the regeneration model. In turning processes, the time delay is basically determined by the rotation of the workpiece but it is also affected by the current and the delayed position of the tool. This results in a DDE with state-dependent delay (SD-DDE), where the delay depends on the present state and also on a delayed state. The analysis of SD-DDEs is a recently developing research area in mathematics (see, e.g., [22-26]).

In engineering practice, DDEs with statedependent delay are rarely used since the appropriate mathematical tools, like linearization techniques, have just been developed recently (see [24, 26]), and these new results have not been adopted in engineering problems yet. Still, the effect of state-dependent delay becomes important in rotary cutting processes (e.g., in milling, or drilling) where the torsional vibrations of the tool are significant in the system's dynamics. Richard et al. [27] and Germay et al. [28] investigated drilling with drag bits and showed that state-dependent regenerative delay arises due to the torsional vibration of the tool. They investigated self-excited vibrations and periodic orbits of the tool numerically. Insperger et al. [29] showed that state-dependent delay arise in the governing equation of the milling process even when only the bending oscillation of the tool is considered and its torsional compliance is neglected. The state-dependency of the regenerative delay due to the bending compliance of the milling tool was also derived by Long et al. [15].

SD-DDEs are always nonlinear, since the state itself arises in its own argument trough the delay. The linearized system, however, is a DDE with constant (or time-dependent) delay. Linearization of SD-DDEs is complicated by the fact that the solution of the system is not differentiable with respect to the state-dependent delay (see, e.g., [30] and the references therein). Consequently, "true" linearization is not possible, rather we are looking for a linear DDE, which is associated to the original system in the sense that they have the same local stability properties. For example, consider the autonomous scalar SD-DDE

$\dot{x}(t)=x\left(t-\left(\tau_{0}+x(t)\right)\right)$.

This is a nonlinear equation due to the state-dependent time delay $\tau(x(t))=\tau_{0}+x(t)$. The DDE

$\dot{y}(t)=y\left(t-\tau_{0}\right)$

with constant time delay is a linear system that can be considered as a linear variational system corresponding to Equation (1) around the equilibrium $x=0$. In our terminology, linearization means that the trivial solutions $y(t) \equiv 0$ of Equation (2) and $x(t) \equiv 0$ of Equation (1) are asymptotically stable at the same time. Linearization techniques for general autonomous SDDDEs were given by Hartung and Turi [24] and for time-periodic SD-DDEs by Hartung [26].

In this paper, a 2 DOF model of turning process is considered. In Section 2, it is shown that an accurate modelling of the regenerative effect results in a state-dependent delay, and the governing equation is an SD-DDE. The linearized equation corresponding to the steady state motion of the tool is determined in Section 3 using the technique of Hartung and Turi [24]. 
It is shown that the associated linearized equation differs from the DDE with constant delay used in the standard turning models. In Section 4, the stability analysis of the system is performed analytically, and it is shown that the incorporation of the state-dependent delay into the model slightly affects linear stability properties of the system.

\section{Mechanical model with state-dependent regenerative delay}

Figure 1 shows the turning process in question. The tool is assumed to be compliant that experiences bending oscillations in directions $x$ and $y$, while the workpiece is assumed to be rigid. Therefore, the system can be modeled as a 2 DOF oscillator that is excited by the cutting force, as it is shown in Fig. 2. The tool is modeled as a beam, and the associated modal parameters are: the mass $m$, the damping $c_{x}$ and $c_{y}$ and the stiffness $k_{x}$ and $k_{y}$. The governing equations read

$$
\begin{aligned}
& m \ddot{x}(t)+c_{x} \dot{x}(t)+k_{x} x(t)=F_{x}, \\
& m \ddot{y}(t)+c_{y} \dot{y}(t)+k_{y} y(t)=-F_{y} .
\end{aligned}
$$

The $x$ and $y$ components of the cutting force can be written as

$$
\begin{aligned}
& F_{x}=K_{x} w h^{q}, \\
& F_{y}=K_{y} w h^{q},
\end{aligned}
$$

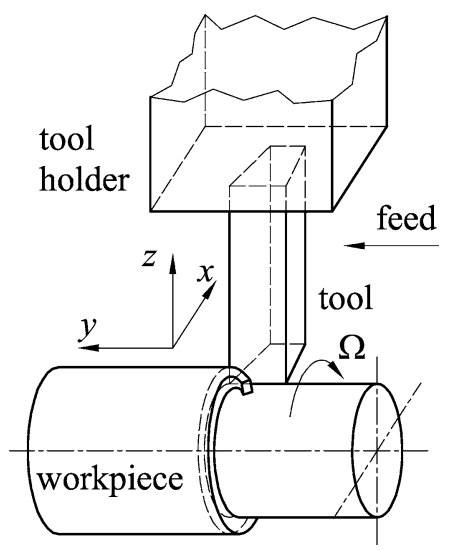

Fig. 1 Turning model where $K_{x}$ and $K_{y}$ are the cutting coefficients in the $x$ and $y$ directions, $w$ is the depth of cut, $h$ is the chip thickness and $q$ is an exponent ( $q=0.75$ is a typical empirical value for this parameter). In this model, it is assumed that the tool never leaves the workpiece, that is, $h>0$ during the cutting process.

The chip thickness $h$ is determined by the feed motion, by the current tool position and by an earlier position of the tool. The time delay $\tau$ between the present and the previous cut is determined by the equation

$R \Omega \tau=2 R \pi+x(t)-x(t-\tau)$,

where $\Omega$ is the spindle speed given in $[\mathrm{rad} / \mathrm{s}]$ and $R$ is the radius of the workpiece. This is an implicit equation for the time delay. It can be seen that the delay actually depends on the current state $x(t)$ and on a delayed state $x(t-\tau)$, that is, the time delay is state-dependent: $\tau\left(x_{t}\right)$, where $x_{t}(s)=x(t+s), s \in[-r, 0], r \in \mathbb{R}^{+}$describes the past of the state.

The chip thickness can be given as the combination of the feed, the present tool position and the delayed position of the tool:

$h=v \tau\left(x_{t}\right)+y(t)-y\left(t-\tau\left(x_{t}\right)\right)$,

where $v$ is the speed of the feed.

Thus, the governing equation can be written as

$$
\begin{aligned}
& m \ddot{x}(t)+c_{x} \dot{x}(t)+k_{x} x(t) \\
& \quad=K_{x} w\left(v \tau\left(x_{t}\right)+y(t)-y\left(t-\tau\left(x_{t}\right)\right)\right)^{q}, \\
& m \ddot{y}(t)+c_{y} \dot{y}(t)+k_{y} y(t) \\
& \quad=-K_{y} w\left(v \tau\left(x_{t}\right)+y(t)-y\left(t-\tau\left(x_{t}\right)\right)\right)^{q} .
\end{aligned}
$$

This is a system of SD-DDEs, where the statedependent delay $\tau\left(x_{t}\right)$ is given by the implicit Equation (7).

Equations (9) and (10) can be written in the compact form

$$
\dot{\mathbf{z}}(t)=\mathbf{f}\left(\mathbf{z}(t), \mathbf{z}\left(t-\tau\left(\mathbf{z}_{t}\right)\right), \tau\left(\mathbf{z}_{t}\right)\right)
$$




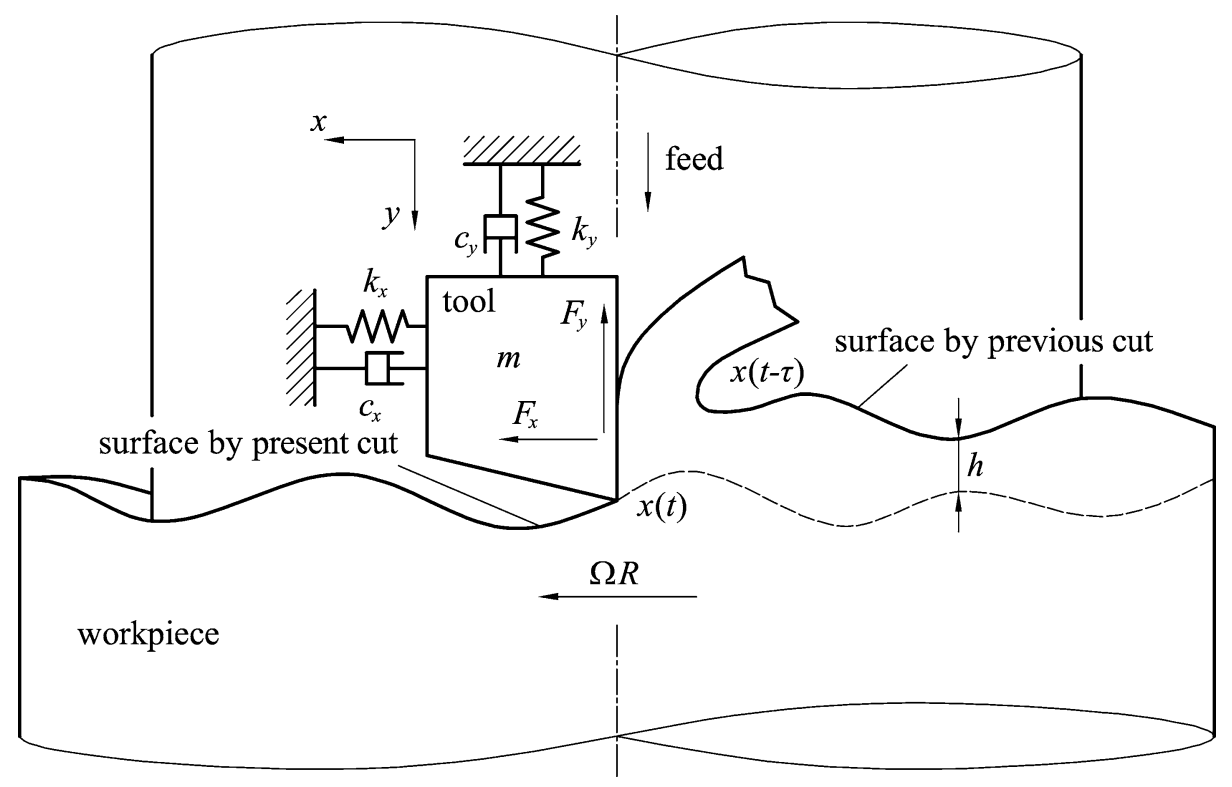

Fig. 2 Model of regeneration in turning process

where

$\mathbf{z}=\left(\begin{array}{c}x \\ y \\ \dot{x} \\ \dot{y}\end{array}\right)$,

$\mathbf{f}=\left(\begin{array}{c}\dot{x} \\ \dot{y} \\ -\frac{c_{x}}{m} \dot{x}-\frac{k_{x}}{m} x+\frac{K_{x} w}{m}\left(v \tau\left(x_{t}\right)+y(t)-y\left(t-\tau\left(x_{t}\right)\right)\right)^{q} \\ -\frac{c_{y}}{m} \dot{y}-\frac{k_{y}}{m} y-\frac{K_{y} w}{m}\left(v \tau\left(x_{t}\right)+y(t)-y\left(t-\tau\left(x_{t}\right)\right)\right)^{q}\end{array}\right)$

and $\mathbf{z}_{t}(s)=\mathbf{z}(t+s), s \in[-r, 0], r \in \mathbb{R}^{+}$. Note that $\mathbf{f}$ also depends explicitly on the delay $\tau\left(\mathbf{z}_{t}\right)=\tau\left(x_{t}\right)$.

\section{Construction of the associated linear system}

For nonlinear systems, a standard way for stability analysis consists of two steps: linearization of the nonlinear system and investigation of the characteristic exponents or characteristic multipliers of the linearized system.

In Hartung and Turi [24] (see Theorem 3.3 on page 423), under very non-restrictive conditions on the SDDDE, it was shown that the asymptotic stability of the trivial solution of the associated linearized equation implies the asymptotic stability of a constant steadystate solution of the original equation. In particular, the linearized system associated to the constant solution $\mathbf{z}(t) \equiv \overline{\mathbf{z}}$ of SD-DDE (11) is given as

$$
\begin{aligned}
\dot{\mathbf{u}}= & \mathrm{D}_{1} \mathbf{f}\left(\overline{\mathbf{z}}, \overline{\mathbf{z}}, \tau\left(\overline{\mathbf{z}}_{t}\right)\right) \mathbf{u}(t)+\mathrm{D}_{2} \mathbf{f}\left(\overline{\mathbf{z}}, \overline{\mathbf{z}}, \tau\left(\overline{\mathbf{z}}_{t}\right)\right) \mathbf{u}(t-\tau(\overline{\mathbf{z}})) \\
& +\mathrm{D}_{3} \mathbf{f}\left(\overline{\mathbf{z}}, \overline{\mathbf{z}}, \tau\left(\overline{\mathbf{z}}_{t}\right)\right) \mathrm{D} \tau\left(\overline{\mathbf{z}}_{t}\right) \mathbf{u}_{t},
\end{aligned}
$$

where $D_{1} \mathbf{f}, D_{2} \mathbf{f}, D_{3} \mathbf{f}$ denotes the derivatives with respect to the 1st, 2nd and 3rd argument of $\mathbf{f}$, respectively, and $\mathrm{D} \tau$ denotes the Frechét derivative (the infinite dimensional gradient) of the time delay $\tau$ with respect to $\mathbf{z}_{t}$. The vector $\mathbf{u}$ reads

$\mathbf{u}=\left(\begin{array}{llll}\xi & \eta & \dot{\xi} & \dot{\eta}\end{array}\right)^{\mathrm{T}}$,

where $\xi$ and $\eta$ denote the perturbations around the constant solution $x(t) \equiv \bar{x}$ and $y(t) \equiv \bar{y}$.

First, the constant solution

$\overline{\mathbf{z}}=\left(\begin{array}{llll}\bar{x} & \bar{y} & 0 & 0\end{array}\right)^{\mathrm{T}}$

of the SD-DDE (11) is determined. This solution corresponds to the stationary cutting process with a constant deflection of the tool. Substitution of $x(t) \equiv \bar{x}$ into Equation (7) gives the constant delay

$\tau\left(\bar{x}_{t}\right)=\bar{\tau}=\frac{2 \pi}{\Omega}+\frac{\bar{x}-\bar{x}}{R \Omega}=\frac{2 \pi}{\Omega}$. 
This constant time delay is used by the standard models of turning processes where the delay is assumed to be determined only by the workpiece rotation.

The substitution of $x(t) \equiv \bar{x}$ and $y(t) \equiv \bar{y}$ into Equations (9) and (10) (or the substitution of $\mathbf{z}(t) \equiv \overline{\mathbf{z}}$ into Equation (11)) gives the solution

$\bar{x}=\frac{K_{x} w(v \bar{\tau})^{q}}{k_{x}}$,

$\bar{y}=-\frac{K_{y} w(v \bar{\tau})^{q}}{k_{y}}$.

This constant steady-state solution is equal to the deflection of the tool for a stationary case, when the tool does not vibrate during the cutting process, but it has a constant deflection.

Now, the terms in Equation (13) are given as

$$
\begin{aligned}
& \mathrm{D}_{1} \mathbf{f}\left(\overline{\mathbf{z}}, \overline{\mathbf{z}}, \tau\left(\overline{\mathbf{z}}_{t}\right)\right) \\
& =\left(\begin{array}{cccc}
0 & 0 & 1 & 0 \\
0 & 0 & 0 & 1 \\
-\frac{k_{x}}{m} & \frac{K_{x} w}{m} q(v \bar{\tau})^{q-1} & -\frac{c_{x}}{m} & 0 \\
0 & -\frac{k_{y}}{m}-\frac{K_{y} w}{m} q(v \bar{\tau})^{q-1} & 0 & -\frac{c_{y}}{m}
\end{array}\right),
\end{aligned}
$$

$\mathrm{D}_{2} \mathbf{f}\left(\overline{\mathbf{z}}, \overline{\mathbf{z}}, \tau\left(\overline{\mathbf{z}}_{t}\right)\right)=\left(\begin{array}{cccc}0 & 0 & 0 & 0 \\ 0 & 0 & 0 & 0 \\ 0 & -\frac{K_{x} w}{m} q(v \bar{\tau})^{q-1} & 0 & 0 \\ 0 & \frac{K_{y} w}{m} q(v \bar{\tau})^{q-1} & 0 & 0\end{array}\right)$

and

$\mathrm{D}_{3} \mathbf{f}\left(\overline{\mathbf{z}}, \overline{\mathbf{z}}, \tau\left(\overline{\mathbf{z}}_{t}\right)\right)=\left(\begin{array}{c}0 \\ 0 \\ \frac{K_{x} w}{m} q(v \bar{\tau})^{q-1} v \\ \frac{K_{y} w}{m} q(v \bar{\tau})^{q-1} v\end{array}\right)$.
The term $\mathrm{D} \tau\left(\overline{\mathbf{z}}_{t}\right) \mathbf{u}_{t}$ is determined in the following way:

$$
\begin{aligned}
\mathrm{D} \tau\left(\overline{\mathbf{z}}_{t}\right) \mathbf{u}_{t}= & \left(\begin{array}{c}
\mathrm{D}_{x_{t}} \tau\left(\overline{\mathbf{z}}_{t}\right) \\
\mathrm{D}_{y_{t}} \tau\left(\overline{\mathbf{z}}_{t}\right) \\
\mathrm{D}_{\dot{x}_{t}} \tau\left(\overline{\mathbf{z}}_{t}\right) \\
\mathrm{D}_{\dot{y}_{t}} \tau\left(\overline{\mathbf{z}}_{t}\right)
\end{array}\right)^{\mathrm{T}}\left(\begin{array}{c}
\xi_{t} \\
\eta_{t} \\
\dot{\xi}_{t} \\
\dot{\eta}_{t}
\end{array}\right) \\
= & \mathrm{D}_{x_{t}} \tau\left(\overline{\mathbf{z}}_{t}\right) \xi_{t}+\mathrm{D}_{y_{t}} \tau\left(\overline{\mathbf{z}}_{t}\right) \eta_{t}+\mathrm{D}_{\dot{x}_{t}} \tau\left(\overline{\mathbf{z}}_{t}\right) \dot{\xi}_{t} \\
& +\mathrm{D}_{\dot{y}_{t}} \tau\left(\overline{\mathbf{z}}_{t}\right) \dot{\eta}_{t},
\end{aligned}
$$

where $\mathrm{D}_{x_{t}} \tau, \mathrm{D}_{y_{t}} \tau, \mathrm{D}_{\dot{x}_{t}} \tau$ and $\mathrm{D}_{\dot{y}_{t}} \tau$ denote the Frechét derivatives of $\tau$ with respect to the $1 \mathrm{st}, 2 \mathrm{nd}, 3 \mathrm{rd}$ and 4 th elements of vector $\mathbf{z}_{t}$. As it can be seen in Equation (7), the time delay depends only on the first element $x_{t}$ of vector $\mathbf{z}_{t}$, consequently, $\mathrm{D}_{y_{t}} \tau=0, \mathrm{D}_{\dot{x}_{t}} \tau=0, \mathrm{D}_{\dot{y}_{t}} \tau=0$ and

$\mathrm{D} \tau\left(\overline{\mathbf{z}}_{t}\right) \mathbf{u}_{t}=\mathrm{D}_{x_{t}} \tau\left(\overline{\mathbf{z}}_{t}\right) \xi_{t}$

Take the Frechét derivative of both sides of Equation (7) with respect to $x_{t}$ :

$R \Omega \mathrm{D} \tau\left(\bar{x}_{t}\right) \xi_{t}=\xi(t)-\xi\left(t-\tau\left(\bar{x}_{t}\right)\right)$.

From here, using the notation $\bar{\tau}=\tau\left(\bar{x}_{t}\right)$, we get

$\mathrm{D} \tau\left(\bar{x}_{t}\right) \xi_{t}=\frac{\xi(t)-\xi(t-\bar{\tau})}{R \Omega}$.

Substitution of Equations (19), (20), (21), (23) and (25) into Equation (13) gives the linearized equation

$$
\frac{\mathrm{d}}{\mathrm{d} t}\left(\begin{array}{c}
\xi \\
\eta \\
\dot{\xi} \\
\dot{\eta}
\end{array}\right)
$$

$$
=\left(\begin{array}{c}
\dot{\xi} \\
\dot{\eta} \\
-\frac{k_{x}}{m} \xi-\frac{c_{x}}{m} \dot{\xi}+\frac{K_{x} w}{m} q(v \bar{\tau})^{q-1}\left(\Delta \eta+\frac{v}{R \Omega} \Delta \xi\right) \\
-\frac{k_{x}}{m} \eta-\frac{c_{x}}{m} \dot{\eta}-\frac{K_{x} w}{m} q(v \bar{\tau})^{q-1}\left(\Delta \eta+\frac{v}{R \Omega} \Delta \xi\right)
\end{array}\right),
$$

where $\Delta \eta=\eta(t)-\eta(t-\bar{\tau})$ and $\Delta \xi=\xi(t)-\xi(t-$ $\bar{\tau})$. 
Transformation of Equation (26) results in the linear system of DDEs

$$
\begin{aligned}
& m \ddot{\xi}(t)+c_{x} \dot{\xi}(t)+k_{x} \xi(t) \\
& =K_{x} w q(v \bar{\tau})^{q-1}((\eta(t)-\eta(t-\bar{\tau})) \\
& \left.\quad+\frac{v}{R \Omega}(\xi(t)-\xi(t-\bar{\tau}))\right), \\
& m \ddot{\eta}(t)+c_{y} \dot{\eta}(t)+k_{y} \eta(t) \\
& =-K_{y} w q(v \bar{\tau})^{q-1}((\eta(t)-\eta(t-\bar{\tau})) \\
& \left.\quad+\frac{v}{R \Omega}(\xi(t)-\xi(t-\bar{\tau}))\right),
\end{aligned}
$$

with constant time delay $\bar{\tau}=2 \pi / \Omega$.

It can easily be seen that if the state-dependency of the delay is not modeled, i.e., $\tau \equiv \bar{\tau}$ is used in the model, then the linearized equations of motion read

$$
\begin{aligned}
& m \ddot{\xi}(t)+c_{x} \dot{\xi}(t)+k_{x} \xi(t) \\
& \quad=K_{x} w q(v \bar{\tau})^{q-1}(\eta(t)-\eta(t-\bar{\tau})), \\
& m \ddot{\eta}(t)+c_{y} \dot{\eta}(t)+k_{y} \eta(t) \\
& \quad=-K_{y} w q(v \bar{\tau})^{q-1}(\eta(t)-\eta(t-\bar{\tau})) .
\end{aligned}
$$

Note that Equation (29) is an ordinary differential equation forced by $\eta$, while Equation (30) is identical to the standard linear model of 1 DOF turning with constant regenerative delay. Consequently, the stability of the constant delay model is determined only by Equation (30).

Comparison of Equations (27)-(28) and (29)-(30) shows that the only difference between the statedependent delay model and the standard model is the term $\frac{v}{R \Omega}(\xi(t)-\xi(t-\bar{\tau}))$. Actually, the appearance of this additional term is related to the fact that the chip thickness and, consequently, the cutting force explicitly depend on the state-dependent time delay as it can be seen in Equation (8) and as it was noted after Equation (12).

\section{Stability analysis}

In the following investigations, it is assumed that the tool is symmetric: $c_{x}=c_{y}=c, k_{x}=k_{y}=k$. The corresponding natural angular frequency is $\omega_{n}=\sqrt{k / m}$ and the damping ratio is $\zeta=c /\left(2 m \omega_{n}\right)$. Introduce the normalized time $\tilde{t}=\omega_{n} t$, and by abuse of notation, drop the tilde immediately. The equations of motion (27) and (28) can be written in the form

$$
\begin{aligned}
\ddot{\xi}(t) & +2 \zeta \dot{\xi}(t)+\xi(t) \\
= & \frac{1}{k_{r}} K_{1} \rho^{q-1}\left(\eta(t)-\eta\left(t-\omega_{n} \bar{\tau}\right)\right) \\
& +\frac{1}{k_{r}} K_{1} \rho^{q}\left(\xi(t)-\xi\left(t-\omega_{n} \bar{\tau}\right)\right), \\
\ddot{\eta}(t)+ & 2 \zeta \dot{\eta}(t)+\eta(t) \\
= & -K_{1} \rho^{q-1}\left(\eta(t)-\eta\left(t-\omega_{n} \bar{\tau}\right)\right) \\
& -K_{1} \rho^{q}\left(\xi(t)-\xi\left(t-\omega_{n} \bar{\tau}\right)\right),
\end{aligned}
$$

where $k_{r}=K_{y} / K_{x}$ is the cutting force ratio, $K_{1}=$ $\left(K_{y} w q(2 \pi R)^{q-1}\right) /\left(m \omega_{n}^{2}\right)$ is the dimensionless depth of cut (or chip width), $\rho=v /(R \Omega)$ is the dimensionless feed per revolution. Note that $\rho=f_{Z} /(2 \pi R)$, where $f_{Z}=v \bar{\tau}$ is the feed per revolution and $2 \pi R$ is the circumference of the workpiece. Since $f_{Z} \ll 2 \pi R$, practically, $\rho \ll 1$.

Stability analysis of the linear autonomous system of DDEs (31)-(32) is performed following the developments in Stépán [5]. First, the characteristic equation is determined, then the stability boundaries in the parameter plane $\left(\Omega / \omega_{n}, K_{1}\right)$ are given, where $\Omega / \omega_{n}$ is the normalized spindle speed.

A lengthy but straightforward calculation gives the characteristic equation of system (31)-(32) in the form

$$
\begin{aligned}
& \left(\lambda^{2}+2 \zeta \lambda+1\right)\left(\lambda^{2}+2 \zeta \lambda+1\right. \\
& \left.\quad+K_{1} \rho^{q-1}\left(1-\frac{\rho}{k_{r}}\right)\left(1-\mathrm{e}^{-\omega_{n} \lambda \bar{\tau}}\right)\right)=0 .
\end{aligned}
$$

There are two roots with negative real parts determined by $\lambda^{2}+2 \zeta \lambda+1=0$, and the remaining roots are determined by the transcendental equation

$$
\begin{aligned}
& \lambda^{2}+2 \zeta \lambda+1+K_{1} \rho^{q-1} \\
& \quad \times\left(1-\frac{\rho}{k_{r}}\right)\left(1-\mathrm{e}^{-\omega_{n} \lambda \bar{\tau}}\right)=0 .
\end{aligned}
$$

Substitution of $\lambda=\mathrm{i} \omega$ and $\bar{\tau}=2 \pi / \Omega$ into Equation (34) and separation of the imaginary and real 
parts give the closed form stability boundaries

$$
\begin{gathered}
K_{1 \mathrm{SDD}}=\left(\frac{k_{r}}{k_{r}-\rho}\right) \frac{\left(\omega^{2}-1\right)^{2}+(2 \zeta \omega)^{2}}{2 \rho^{q-1}\left(\omega^{2}-1\right)}, \\
\frac{\Omega}{\omega_{n}}=\frac{\omega \pi}{\arctan \left(\frac{1-\omega^{2}}{2 \zeta \omega}\right)+k \pi}, \quad k=1,2, \ldots,
\end{gathered}
$$

where the chatter frequency $\omega$ is used as a parameter, and the index SDD refers to state-dependent delay.

Consider now the turning model with constant regenerative delay described by Equation (30). The corresponding characteristic equation reads

$$
\lambda^{2}+2 \zeta \lambda+1+K_{1} \rho^{q-1}\left(1-\mathrm{e}^{-\omega_{n} \lambda \bar{\tau}}\right)=0 .
$$

Comparison to Equation (34) shows that the only difference due to the state dependency of the delay is the appearance of the multiplicative term $\left(1-\frac{\rho}{k_{r}}\right)$. The stability boundaries corresponding to the model with constant delay (that actually coincides with the case of the standard 1 DOF turning case) is determined by

$K_{1 \mathrm{CD}}=\frac{\left(\omega^{2}-1\right)^{2}+(2 \zeta \omega)^{2}}{2 \rho^{q-1}\left(\omega^{2}-1\right)}$,

while the expression for $\Omega / \omega_{n}$ is identical to Equation (36).

This shows that if the state dependency of the time delay is included into the model, then the resulting stability boundaries are shifted upwards by the ratio

$$
\frac{K_{1 \mathrm{SDD}}}{K_{1 \mathrm{CD}}}=\frac{k_{r}}{k_{r}-\rho},
$$

where $K_{1 \mathrm{SDD}}$ and $K_{1 \mathrm{CD}}$ denotes critical depth of cuts for state-dependent and constant delay models, respectively. Practically, this ratio is close to 1 , since $\rho \ll 1$ as it was noted after Equation (32), and a typical value of the cutting force ratio is $k_{r}=0.3$. This shows that the state-dependency of the time delay is important if $\rho$ is large, i.e, large feed is applied for a workpiece with small diameter.

In Fig. 3, stability charts are shown for different dimensionless feeds $\rho$ per revolution both for state-dependent and constant delay models. It can be seen that stability boundaries are higher for the statedependent delay model than those of the constant delay model. This can also be seen from Equation (39), since
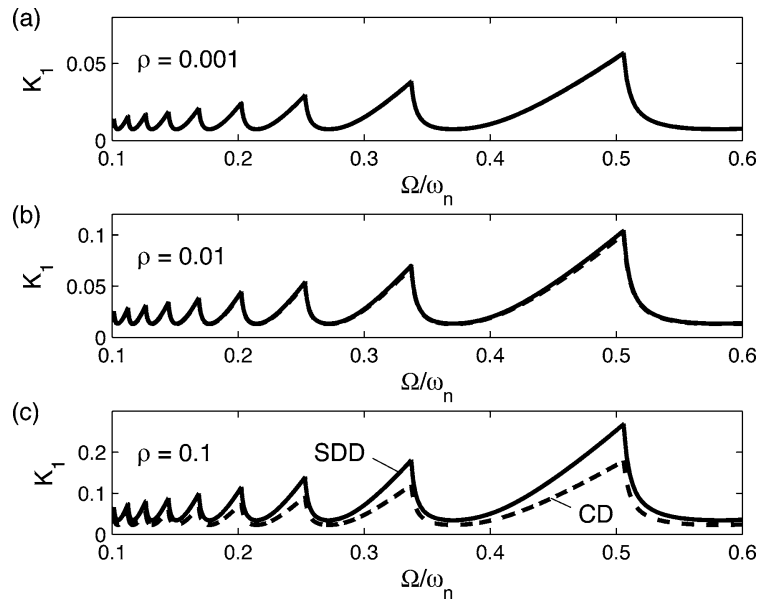

Fig. 3 Stability charts for different dimensionless feeds $\rho$ per revolution. Continuous and dashed lines correspond to statedependent and constant delay models. The parameters are $\zeta=$ $0.02, q=0.75$ and $k_{r}=0.3$

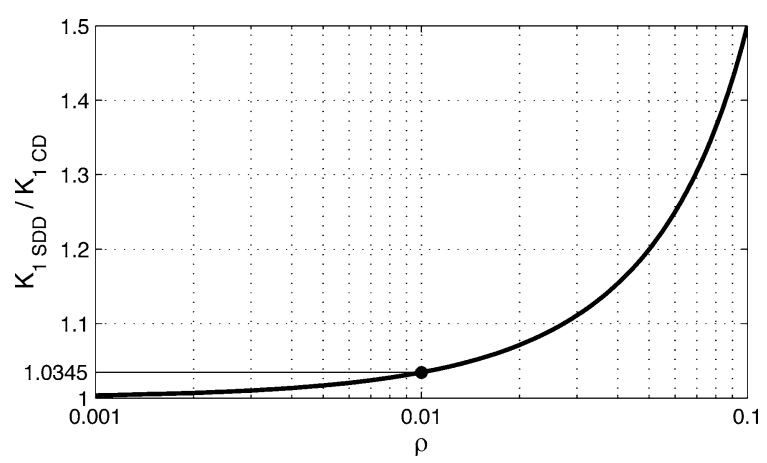

Fig. 4 Ratio of the critical depths of cut for state-dependent and constant delay models as the function of the dimensionless feed $\rho$ per revolution $\left(k_{r}=0.3\right)$

$K_{1 \mathrm{SDD}} / K_{1 \mathrm{CD}}>1$ for positive $k_{r}$ and $\rho$. The difference between the two models increases with increasing parameter $\rho$.

In Fig. 4 , the ratio $K_{1 \mathrm{SDD}} / K_{1 \mathrm{CD}}$ is shown as a function of $\rho$. The case $\rho=0.01$ can be considered as a limit case in practice for turning: when a $6.4 \mathrm{~mm}$ diameter workpiece is cut with $0.2 \mathrm{~mm}$ feed per revolution, the stability boundary is shifted upwards by $3.45 \%$ relative to the constant delay model. Note that this difference might be even larger for some other manufacturing operations, like milling (see [29]) or drilling with high torsional compliance of the tool (see [27, 28]). 


\section{Conclusion}

A 2 DOF model of turning process was considered. It was shown through an accurate model of the surface regeneration that the regenerative delay is determined by the combination of the workpiece rotation and the tool vibrations resulting in a state-dependent time delay. The corresponding equation is a delay-differential equation with state-dependent delay as opposed to the standard models with constant time delay.

The linear equation corresponding to the stationary cutting with constant deflection of the tool was derived using the technique of Hartung and Turi [24]. It was shown that the resulting linearized equation is different from the DDE with constant time delay used in standard turning models. An additional term arises due to the explicit dependence of the cutting force on the statedependent delay. Stability analysis of the linearized system was performed analytically, and it was shown that the incorporation of the state-dependent delay into the model slightly affects linear stability properties of the system in the practical parameter domains. The influence of the state-dependent delay may be even more significant on the nonlinear behavior of the system.

Although the importance of the state-dependent delay model seems to be limited at the moment, it might become more relevant in the future, similarly to the development of the milling models, where the timeperiodicity of the cutting force had been neglected in the past, and its importance (that it may cause period doubling vibrations) was realized only with the recent appearance of high-speed milling technology.

Acknowledgements This research was supported in part by the János Bolyai Research Scholarship of the Hungarian Academy of Sciences, and by the Hungarian National Science Foundation under grant no. OTKA F047318 and OTKA T043368.

\section{References}

1. Tlusty, J., Polacek, A., Danek, C., Spacek, J.: Selbsterregte Schwingungen an Werkzeugmaschinen. VEB Verlag Technik, Berlin (1962)

2. Tobias, S.A.: Machine Tool Vibration. Blackie, London (1965)

3. Balachandran, B., Zhao, M.X.: A mechanics based model for study of dynamics of milling operations. Meccanica 35(2), 89-109 (2000)

4. Peigne, G., Paris, H., Brissaud, D., Gouskov, A.: Impact of the cutting dynamics of small radial immersion milling operations on machined surface roughness. Int. J. Mach. Tools Manuf. 44(11), 1133-1142 (2004)

5. Stépán, G.: Retarded Dynamical Systems. Longman, Harlow (1989)

6. Altintas, Y., Budak, E.: Analytical prediction of stability lobes in milling. Ann. CIRP 44(1), 357-362 (1995)

7. Insperger, T., Mann, B.P., Stépán, G., Bayly, P.V.: Stability of up-milling and down-milling, Part 1: Alternative analytical methods. Int. J. Mach. Tools Manuf. 43(1), 25-34 (2003)

8. Bayly, P.V., Halley, J.E., Mann, B.P., Davies, M.A.: Stability of interrupted cutting by temporal finite element analysis. J. Manuf. Sci. Eng. 125(2), 220-225 (2003)

9. Faassen, R.P.H., van de Wouw, N., Oosterling, J.A.J., Nijmeijer, H.: Prediction of regenerative chatter by modeling and analysis of high-speed milling. Int. J. Mach. Tools Manuf. 43(14), 1437-1446 (2003)

10. Szalai, R., Stépán, G.: Stability boundaries of highspeed milling corresponding to period doubling are essentially closed curves. Proceedings of ASME International Mechanical Engineering Conference and Exposition, Washington D.C., USA, paper no. IMECE2003-42122 (2003)

11. Corpus, W.T., Endres, W.J.: Added stability lobes in machining processes that exhibit periodic time variation - Part 1 : An analytical solution. J. Manuf. Sci. Eng. 126(3), 467-474 (2004)

12. Merdol, S.D., Altintas, Y.: Multi frequency solution of chatter stability for low immersion milling. J. Manuf. Sci. Eng. 126(3), 459-466 (2004)

13. Gradišek, J., Kalveram, M., Insperger,T., Weinert, K., Stépán, G., Govekar, E., Grabec, I.: On stability prediction for milling. Int. J. Mach. Tools Manuf. 45(7-8), 769-781 (2005)

14. Long, X.-H., Balachandran, B.: Milling model with variable time delay. Proceedings of the 2004 ASME International Mechanical Engineering Congress and Exposition, Anaheim, CA, paper no. IMECE2004-59207 (2004)

15. Long, X.-H., Balachandran, B., Mann, B.P.: Dynamics of milling processes with variable time delay. Nonlinear Dyn., in this issue (2006)

16. Faassen, R., van de Wouw, N., Oosterling, H., Nijmeijer, H.: Updated tool path modelling with periodic delay for chatter prediction in milling. Fifth EUROMECH Nonlinear Dynamics Conference, ENOC 2005, Eindhoven, The Netherlands, pp. 1080-1089 (2005)

17. Sexton, J.S., Milne, R.D., Stone, B.J.: A stability analysis of single point machining with varying spindle speed. Appl. Math. Modelling 1, 310-318 (1977)

18. Jayaram, S., Kapoor, S.G., DeVor, R.E.: Analytical stability analysis of variable spindle speed machining. J. Manuf. Sci. Eng. 122(3), 391-397 (2000)

19. Insperger, T., Stépán, G.: Stability analysis of turning with periodic spindle speed modulation via semi-discretization. J. Vib. Control 10(12), 1835-1855 (2004)

20. Insperger, T., Stépán, G.: Semi-discretization method for delayed systems. Int. J. Numer. Methods Eng. 55(5), 503-518 (2002)

21. Insperger, T., Stépán, G.: Updated semi-discretization method for periodic delay-differential equations with discrete delay. Int. J. Numer. Methods Eng. 61(1), 117-141 (2004) 
22. Győri, I., Hartung, F.: On the exponential stability of a statedependent delay equation. Acta Sci. Math. 66, 71-84 (2000)

23. Krisztin, T., Arino, O.: The 2-dimensional attractor of a differential equation with state-dependent delay. J. Dyn. Differ. Equ. 13, 453-522 (2001)

24. Hartung, F., Turi, J.: Linearized stability in functionaldifferential equations with state-dependent delays. Proceedings of the conference Dynamical Systems and Differential Equations, added volume of Discrete and Continuous Dynamical Systems, pp. 416-425 (2000)

25. Luzyanina, T., Engelborghs, K., Roose, D.: Numerical bifurcation analysis of differential equations with state-dependent delay. Int. J. Bifurcation Chaos 11(3), 737-753 (2001)

26. Hartung, F.: Linearized stability in periodic functional differential equations with state-dependent delays J. Comput. Appl. Math. 174, 201-211 (2005)
27. Richard, T., Germay, C., Detournay, E.: Self-excited stickslip oscillations of drill bits. C. R. Mec. 332(8), 619-626 (2004)

28. Germay, C., van de Wouw, N., Sepulchure, R., Nijmeijer, H.: Axial stick-slip limit cycling in drill-string dynamics with delay, Fifth EUROMECH Nonlinear Dynamics Conference, ENOC 2005, Eindhoven, The Netherlands pp. 1136-1143 (2005)

29. Insperger, T., Stépán, G., Hartung, F., Turi, J.: Statedependent regenerative delay in milling processes. in Proceedings of ASME International Design Engineering Technical Conferences, Long Beach CA (2005), paper no. DETC2005-85282 (2005)

30. Hartung, F., Turi, J.: On differentiability of solutions with respect to parameters in state-dependent delay equations. J. Differ. Equ. 135(2), 192-237 (1997) 